\title{
A call for consensus in defining efficacy in clinical trials for opioid addiction: combined results from a systematic review and qualitative study in patients receiving pharmacological assisted therapy for opioid use disorder
}

Brittany B. Dennis ${ }^{1,2}$, Nitika Sanger ${ }^{2}$, Monica Bawor ${ }^{1}$, Leen Naji ${ }^{3}$, Carolyn Plater ${ }^{4}$, Andrew Worster ${ }^{2,5}$, Julia Woo ${ }^{6}$, Anuja Bhalerao ${ }^{6}$, Natasha Baptist-Mohseni ${ }^{4}$, Alannah Hillmer ${ }^{4}$, Danielle Rice ${ }^{7,8}$, Kim Corace $^{9,10}$, Brian Hutton ${ }^{11,12}$, Peter Tugwell ${ }^{13}$, Lehana Thabane $e^{2,14,15}$ and Zainab Samaan ${ }^{2,4,16^{*}}$ (ID

\begin{abstract}
Background: Given the complex nature of opioid addiction treatment and the rising number of available opioid substitution and antagonist therapies (OSAT), there is no 'gold standard' measure of treatment effectiveness, and each successive trial measures a different set of outcomes which reflect success in arbitrary or opportune terms. We sought to describe the variation in current outcomes employed across clinical trials for opioid addiction, as well as determine whether a discrepancy exists between the treatment targets that patients consider important and how treatment effectiveness is measured in the literature.
\end{abstract}

Methods: We searched nine commonly used databases (e.g., EMBASE, MEDLINE) from inception to August 1, 2015. Outcomes used across trials were extracted and categorized according to previously established domains. To evaluate patient-reported goals of treatment, semi-structured interviews were conducted with 18 adults undergoing methadone treatment.

Results: We identified 60 trials eligible for inclusion. Once outcomes were categorized into eight broad domains (e.g., abstinence/substance abuse), we identified 21 specific outcomes with furthermore 53 subdomains and 118 measurements. Continued opioid use and treatment retention were the most commonly reported measures (46\%, $n=$ 28). The majority of patients agreed that abstinence from opioids was a primary goal in their treatment, although they also stressed goals under-reported in clinical trials.

Conclusions: There is inconsistency in the measures used to evaluate the effectiveness of OSATs. Individual and population level decision making is being guided by a standard of effect considered useful to researchers yet in direct conflict with what patients deem important.

Trial registration: PROSPERO, CRD42013006507.

Keywords: Opioid addiction, Clinical trials, Efficacy, Methodology, Patient important outcomes, Treatment effectiveness

\footnotetext{
*Correspondence: samaanz@mcmaster.ca

${ }^{2}$ Department of Health Research Methods, Evidence, and Impact, McMaster

University, Hamilton, Canada

${ }^{4}$ Department of Psychiatry and Behavioural Neurosciences, McMaster

University, 1280 Main Street West, Hamilton, Ontario L8S 4L8, Canada

Full list of author information is available at the end of the article
}

(c) The Author(s). 2020 Open Access This article is distributed under the terms of the Creative Commons Attribution 4.0 International License (http://creativecommons.org/licenses/by/4.0/), which permits unrestricted use, distribution, and reproduction in any medium, provided you give appropriate credit to the original author(s) and the source, provide a link to the Creative Commons license, and indicate if changes were made. The Creative Commons Public Domain Dedication waiver (http://creativecommons.org/publicdomain/zero/1.0/) applies to the data made available in this article, unless otherwise stated. 


\section{Background}

Information retrieved from the highest quality evidencemost often from randomized controlled trials (RCTs) - is used to inform health care decisions at individual and population levels. From the development of research questions to decisions regarding "significant" treatment targets, the research community exerts a strong influence on the generation of evidence. The end users of this evidencewhether this be physicians, policy makers, or patients-rely on the expert opinion of researchers to design studies and ultimately trust they select the appropriate outcomes to reflect treatment success. Despite best interests, the value of many pharmacological interventions is commonly evaluated on their observed effect across different biochemical and surrogate measurements [1]. Frequently these measurements neither reflect nor acknowledge the values and preferences of the populations they are meant to serve. Patientimportant outcomes reflect the health concerns, fears regarding adverse drug reactions, treatment goals, and overall values of patient populations. These outcomes are often underrepresented in comparison to biologic measurements closely associated with the physiologic disease process [1]. For instance, the majority of trials within the diabetes literature include primary endpoints such as blood glucose level as an indicator of efficacy due to its direct relation to the pathophysiology of diabetes. Outcomes such as death, stroke, infection, pain function, or delayed wound healing have significant impact on patients' lives, yet are often underreported [2]. Unfortunately, patient-important outcomes are often neglected in trials aimed to establish treatment benefit; this deficit is of substantial concern to the growing evidence base in opioid addiction, known formally as opioid use disorder (OUD) [3].

Given the complex nature of OUD treatment and the rising number of available pharmacological opioid substitution and antagonist therapies (OSAT), there is no "gold standard" measure of treatment effect and each successive trial measures a different set of treatment outcomes which reflect success in arbitrary or opportune terms [4-6]. Commonly included endpoints comprise attrition rates, illicit substance use, presence of medical and psychiatric comorbidity, social function as measured by current housing arrangements, collective neighborhood income, educational achievement, employment, and involvement in criminal activity [4-6]. The variation in the selection of outcomes as well as the marked range of definitions, instruments, and measurements of specific outcomes demand the need for further research to establish a summary of the current outcomes utilized in the literature, as well as determine which outcomes reflect patients' values and preferences for the end goals of addiction treatment.

In the current study, we sought to outline the current outcomes employed in clinical trials for opioid addiction, as well as to determine whether a divide exists between the treatment targets patients consider important and those selected to evaluate efficacy in the literature.

\section{Methods}

This study was completed in two phases. In the first phase of the study we completed a systematic review which aimed to describe outcomes used in the current literature to establish effectiveness of different OSATs. The second phase aimed to determine patient's perspectives of successful addiction treatment with emphasis on the patient's end-goals of therapy. Phase 1 of this study used the previously published protocol for a systematic review and network metaanalysis comparing OSAT interventions for OUD during which we also extracted the listing of outcomes reported within each study [7]. The literature search was completed in August 2015; this was not updated for the current study given that the emphasis is not on establishing a superior therapy for addiction, but rather to provide a summary of the outcome measures employed across clinical trials comprising the main body of evidence.

\section{Phase 1: systematic review to establish outcomes used in the current literature Methodology}

The collective body of evidence for OSAT trials was identified using results from a previous systematic review. A summary of the methods for this work are described in the published protocol [7]. The original systematic review utilized for this study was registered in the PROSPERO database (CRD42013006507) and adheres to the PRISMA guidelines [8].

Studies included in the previously published review were limited to trials evaluating pharmacological therapies for opioid addiction in general addiction populations; any studies in special populations, including prison, were excluded. No studies were eliminated based on outcome selection. All primary investigators listed on the NIH Clinical Trial Registry from eligible studies identified during the title screening were contacted for inquires regarding any publications resulting from their trials. The original review placed no constraints on language or date of publication. Animal studies and incomplete studies (pilot, preliminary reports) were excluded. Methodological quality assessment was conducted using the Cochrane Risk of Bias Tool for RCTs.

\section{Summary of outcomes used across OSAT trials}

The primary aim of the current study was to summarize all outcome domains and subdomains and their definitions and outcomes measurements/instruments used for each outcome in trials of OSAT for OUD. Data extraction forms were constructed and pilot tested for use in this review. We abstracted the sample size, mean age, eligibility criteria, intervention description, dose, approaches to missing data, 
outcome definition, outcome measurement, covariates included in regression models if adjusted analyses were performed, and the statistical association reported (e.g., odds ratio (OR), relative risk (RR)).

To provide an organized summary, we structured outcomes into broader categories according to the domains proposed by commonly used measurement scales evaluating addiction severity (i.e., the Addiction Severity Index (ASI) [9] and Maudsley Addiction Profile (MAP)) [10]. These tools evaluate treatment response using the broader domains of substance use behavior, physical and mental health, and social functioning $[9,10]$. Both tools are practical and provide a global assessment of patients' physical and social functioning. Our outcome domains included physical health, psychiatric health and symptoms, abstinence and substance use behavior, and personal and social functioning. Some studies used additional outcomes that did not conform to these domains; thus, we included global quality of life and addiction severity assessments (including global addiction severity, intervention adherence, acceptance of intervention, and resource utilization (e.g., hospital admission) as additional domains. This categorization of outcome domains and subdomains provides researchers and clinicians with an overview of the current outcomes used to assess patients' responses to OSAT.

All outcomes used across trials included in this review were extracted and categorized according to the above described criteria.

\section{Phase 2: qualitative interviewing of patients on pharmacological treatment for OUD Recruitment and interview methodology}

Patients were recruited from two opioid addiction treatment centers in Ontario, Canada using purposive sampling. The research collaborative between the Population Genomics Program at McMaster University and the Canadian Addiction Treatment Centers (CATC) provided a framework for study recruitment, data collection, data analysis, and follow-up. Eligibility criteria included: patients $\geq 18$ years, currently receiving an opioid substitution therapy including methadone maintenance treatment or buprenorphine, able to understand and speak English, and able to provide informed consent [11]. The Hamilton Integrated Research Ethics Board (HiREB) approved this study (HiREB study ID 0168). This study adheres to the STROBE guidelines [12].

Qualitative methods were used to establish patients' perspectives of successful addiction treatment. Structured open-ended interviews were conducted to explore each patient's end-goals of therapy. These interviews identified common themes with regard to addiction treatment goals. The interviews were transcribed and analyzed for themes, clarifications, and deeper understanding of the topics outlined above [11].
Convenience sampling was utilized between two addiction treatment clinics. Recruiting from two separate sites allowed for a broader patient demographic to be covered, as socioeconomic status and homelessness rates were known to differ between sites. Flyers advertising the study were posted at both clinical sites. All patients eligible for recruitment were also approached and informed about the study objectives by the clinic's healthcare staff. Upon gaining informed consent, patients were given a demographic questionnaire and interviewed by two investigators using structured questions and openended questions. No one else was present at these interviews. All patients included in the study were given a $\$ 5$ gift card at the end of the interview [11].

Interviews were conducted by an addiction specialist nurse, Carolyn Platter (BScNurs), and two female research coordinators, Julia Woo (BHSc) and Anuja Bhalerao (BHSc). These team members have performed hundreds of interviews in this population since working with the McMaster GENOA research collaborative. The interviewers were selected in efforts to minimize potential bias generated during data collection. These team members had no previous stake in the research question or design of this study. All interviewers underwent ethics and sensitivity training prior to meeting the patients, as per McMaster University Research Ethics Board Guidelines. Each team member has completed the Tri-Council Policy Statement course. The patients recruited into the study had not been previously interviewed by the team members and we are confident there was no relationship between participants and interviewers prior to the interview. Participants were briefed as to the goals of the study, particularly our aim of establishing whether current research accurately reflects what they wish to gain from treatment.

Interviews were completed using a structured piloted questioning tool with prompts; patients were approached allowing for open-ended answers. Each interview was audio recorded for later transcription. Each interviewer also made field notes, which were used to aid in later transcription. Each interview transcript was carefully investigated for insight into the major research question, "How would you measure success in methadone maintenance or buprenorphine treatment?" We also provided patients a list of commonly anticipated treatment goals and asked them to rank which aspect of recovery meant the most to their addiction treatment. Patients were allowed to rank up to four items. The list provided a summary of different potential goals across substance abuse, physical health, emotional stability, and personal functioning domains. A register of these goals in addition to the interview tool can be found in Additional file 1 . The interviews lasted approximately $40 \mathrm{~min}$ and were conducted on site at the treatment facility between 
September 2015 and February 2016. Interviews were conducted until responses to the major research questions were saturated, having no new themes emerge [11]. Patients were not provided transcribed copies of their interview.

\section{Analysis}

Interviews were transcribed and evaluated for the common definitions of success in addiction treatment as well as aspects of recovery patients found important. Two primary interviewers $(A B, J W)$ were responsible for coding the data, unaided by software. This process was later reviewed by all members of the team. These responses were coded according to the broader domains proposed by popular measurement scales evaluating addiction severity: the ASI [9] and MAP [10]. Additional domains not included in the MAP or ASI were also added. These domains included global quality of life and addiction severity assessments (including global addiction severity measure scores), intervention adherence, acceptance of intervention, and resource utilization (e.g., hospital admission) [11].

Due to the small size and limited power of our sample, no statistical tests were conducted in reference to significant differences between the participants at the two sites [11].

\section{Results}

\section{Phase I: findings from the systematic review}

An annotated flow diagram of the study selection process is presented in Fig. 1. We searched databases since inception to August 1, 2015 and identified 6077 articles. We identified 60 trials with a combined participant sample of 13,341 patients eligible for full text extraction [13-72]. A summary of the included trials is available in Additional file 1. Table 1 summarizes the outcome domains and subdomains used across trials included in this study; the outcomes are categorized into

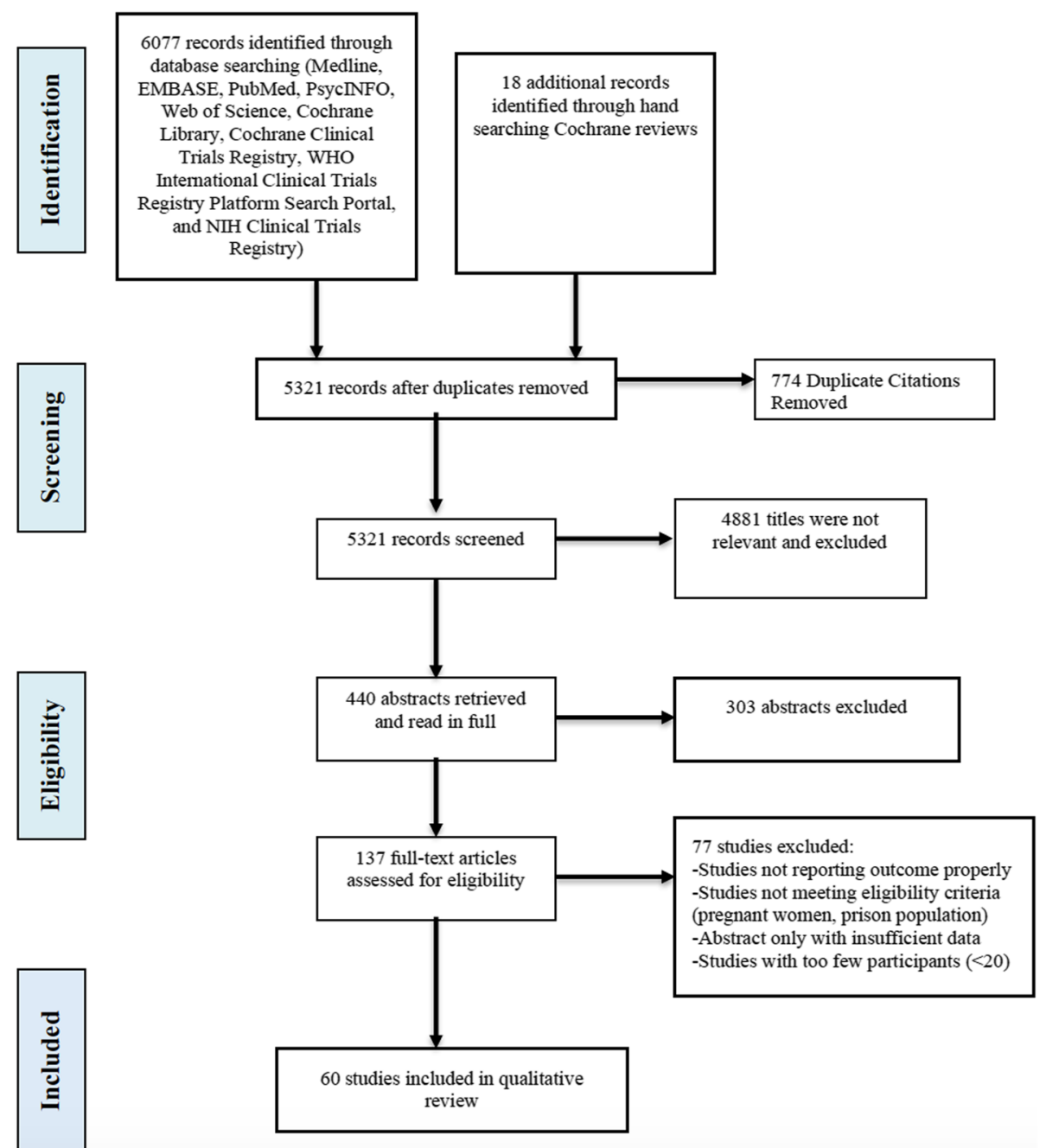

Fig. 1 The preferred reporting items for systematic reviews and meta-analyses (PRISMA) flow diagram 
broad domains, outcome domains, subdomains, and the specific measurements. Within the eight broadest domains (abstinence and substance use behavior, physical health, psychiatric health and symptoms, personal and social functioning, resource utilization, intervention adherence, intervention acceptance, and global quality of life and addiction severity) are 21 more specific outcome domains (e.g., illicit opioid use, illicit non-opioid substance use), and across these outcomes there exist 53 separate definitions or measurements.

Of the 60 trials eligible for inclusion in this review, retention in treatment was the most commonly measured and reported outcome. Of the 28 studies reporting retention in treatment as their primary outcome, 16 different interventions were evaluated. The second most commonly reported outcome was illicit opioid use, which took 17 definitions and a further eight variations in measurement. The wideranging definitions for illicit opioid use included 1) the frequency of use in the form of the mean number or days of use or the percentage of positive urine screens, 2) the mean time patients remain abstinent on therapy or time until the first positive opioid urine screen is observed, 3 ) the number of participants per treatment arm who fulfill a predefined criteria for "success" or "failure" according to their opioid use consumption patterns, and 4) the global severity of opioid use as scored from a validated tool. Further variations arose based on the measurement of opioid use, which included urine toxicology screening with directly observed or non-observed sampling, toxicology screening with hair samples, validated addiction severity measurement tools, as well as weekly activity summaries or self-report.

General physical health outcomes comprised the largest differences in both conceptualization and measurement. Physicians' perception of disease, cardiac function, immune system function, pain severity, and the presence of physical comorbidity were among the commonly measured aspects of general physical health.

\section{Phase II: qualitative interviewing of patients on pharmacological treatment for opioid addiction}

A total of 18 individuals from two treatment centers participated in this study. Sixteen of the participants were currently undergoing MMT at the time of recruitment and two participants were receiving buprenorphine but had received MMT at least one year prior. The mean age of the participants was 36.11 (standard deviation $=10.01$ ) years with majority female $(67 \%)$ and of Caucasian ethnicity (89\%). Participants in one site had a higher mean income $(\$ 48,750$ vs $\$ 35,000)$ and were more likely to be employed (63\% vs $40 \%$ ) compared to the second site's participants, which is expected as the second site was selected purposefully to be economically different. All participants were interviewed in a single session and no repeated sessions were necessitated during the course of this study.

\section{Qualitative interview}

The majority of participants (61.1\%) identified their main goal of methadone treatment as being abstinence from drugs. This goal was clearly indicated by patients, including statements like: "Just being completely off of drugs. To never touch drugs again." Close to a third of these individuals had a more specific goal of being off of methadone completely (38\%). One participant stated specifically (as seen in the following direct quote) that even though they are sober, their ultimate goal is to be "clean" from all opioids.

"When someone tells me I'm not sober because I'm on methadone. I tell them I may not be clean because I'm putting this medication in my body but I am sober. I want to be clean. To me, I'm sober right now, I have been sober for two and a half years. I haven't touched the drugs for two and a half years. At the end of it, I want to be off the methadone completely but I want to be able to taper down till I no longer need it anymore and I want to look back and say that was just a phase in my life. I took the necessary steps to make myself better and I accomplished that. And all the things that I accomplished being on methadone too. So yeah, I just want to get off of it completely, eventually"

Others did not desire to be off methadone and specified methadone was helping them. One participant's main goal was for pain control and not to be off methadone, as it helped them function and be able to move. When asked if they were hoping to get completely off methadone they responded saying, "I don't know if I ever will. I see my doses being reduced but until my health problems are resolved, I have absolutely no problem being on it if it has to be for the rest of my life."

Other goals of methadone treatment that were not as common included being able to get back to their usual lives and able to maintain it, to not be sick, and to manage addictions not only related to drugs but in other domains of their life. Participant's verbatim responses are summarized in Table 2. The percentages presented above reflect an assessment of patient responses presented in Table 2.

\section{Response to predetermined treatment goals}

Seventeen out of the 18 participants completed the section indicating which aspect of recovery meant the most to their addiction treatment. Please refer to Fig. 2 for a graphical summary of patients' first ranked treatment goals. This graphical summary was generated using the individual patient data reported in Table 3, whereby the 


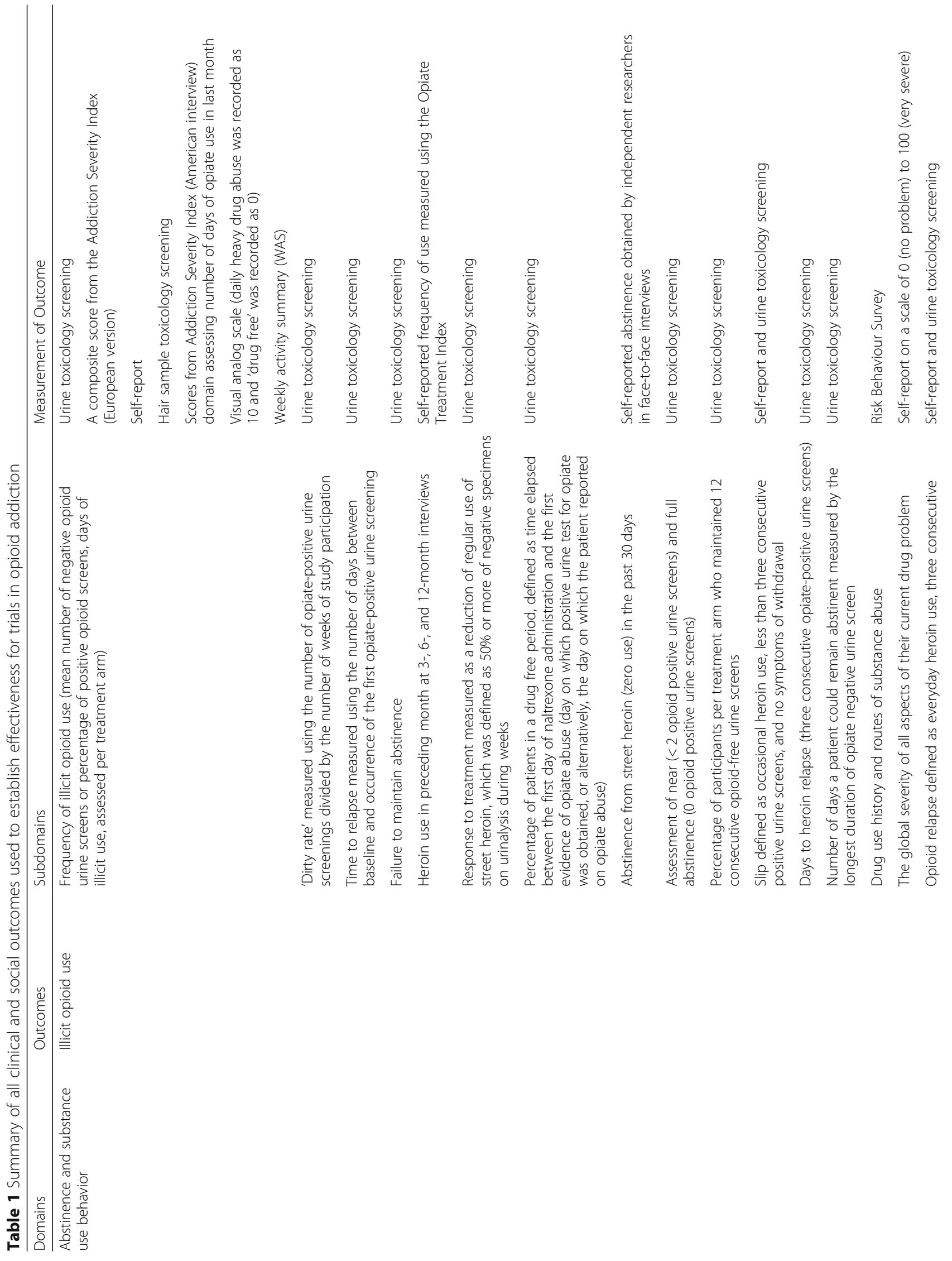




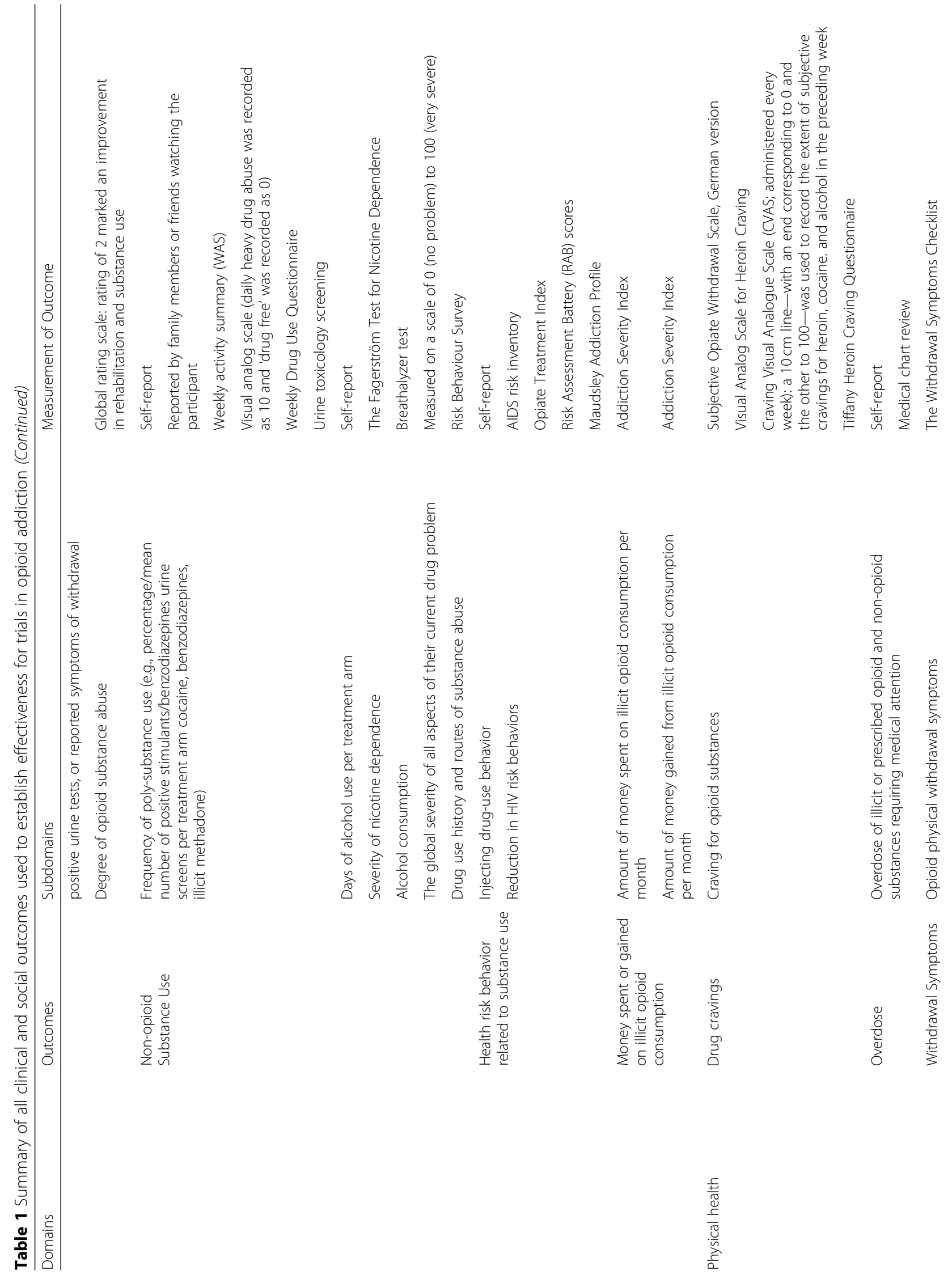




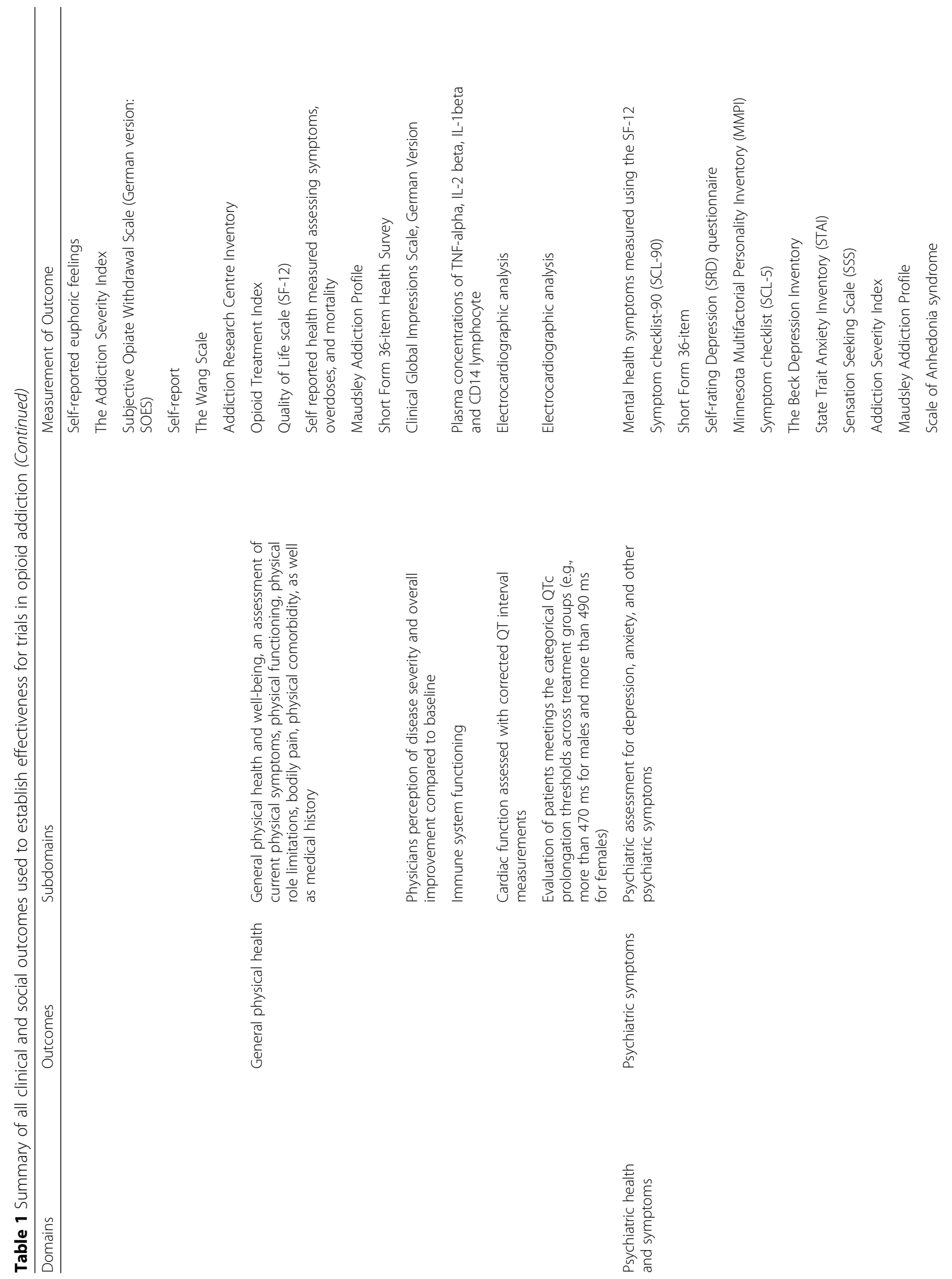




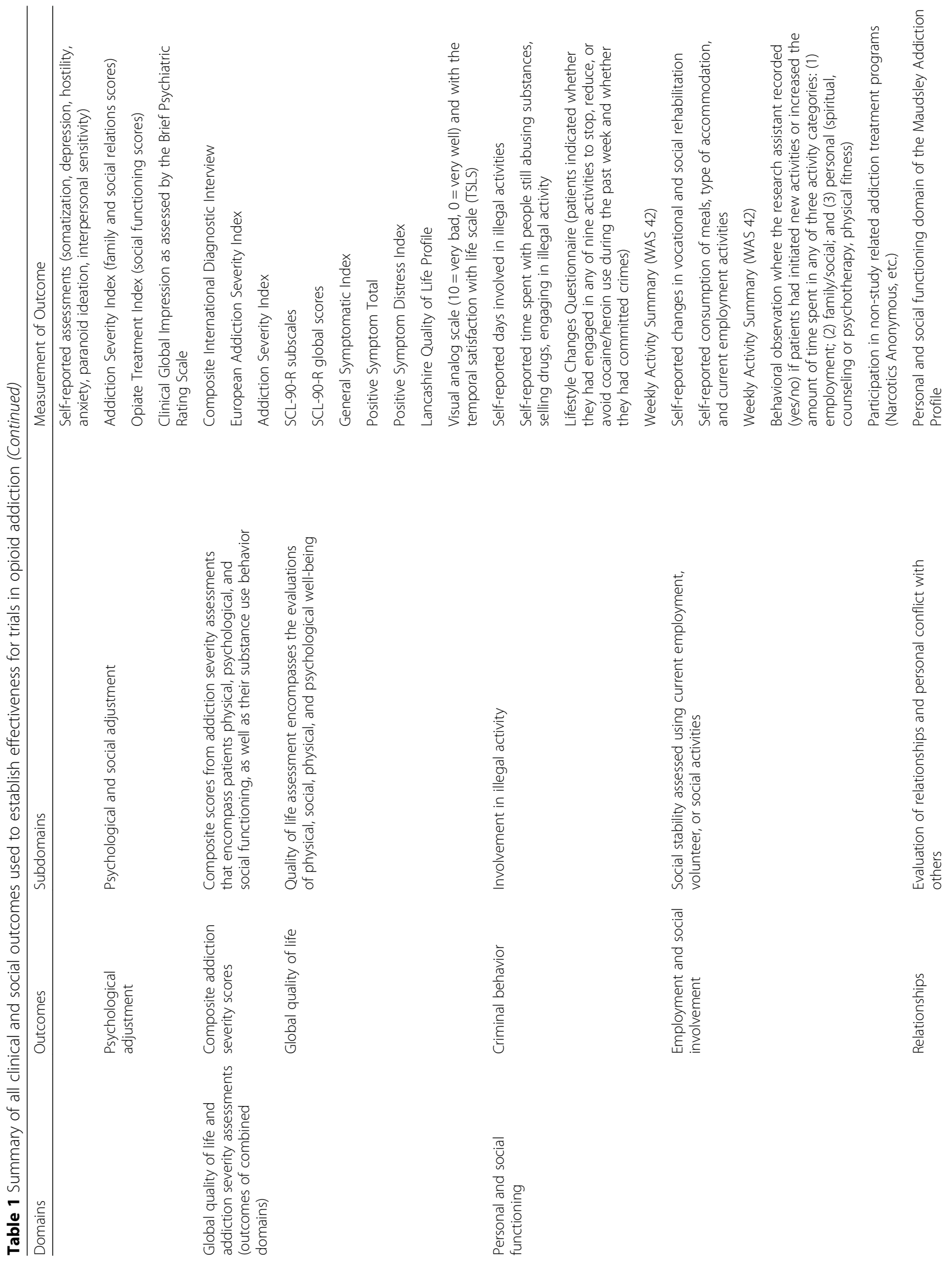




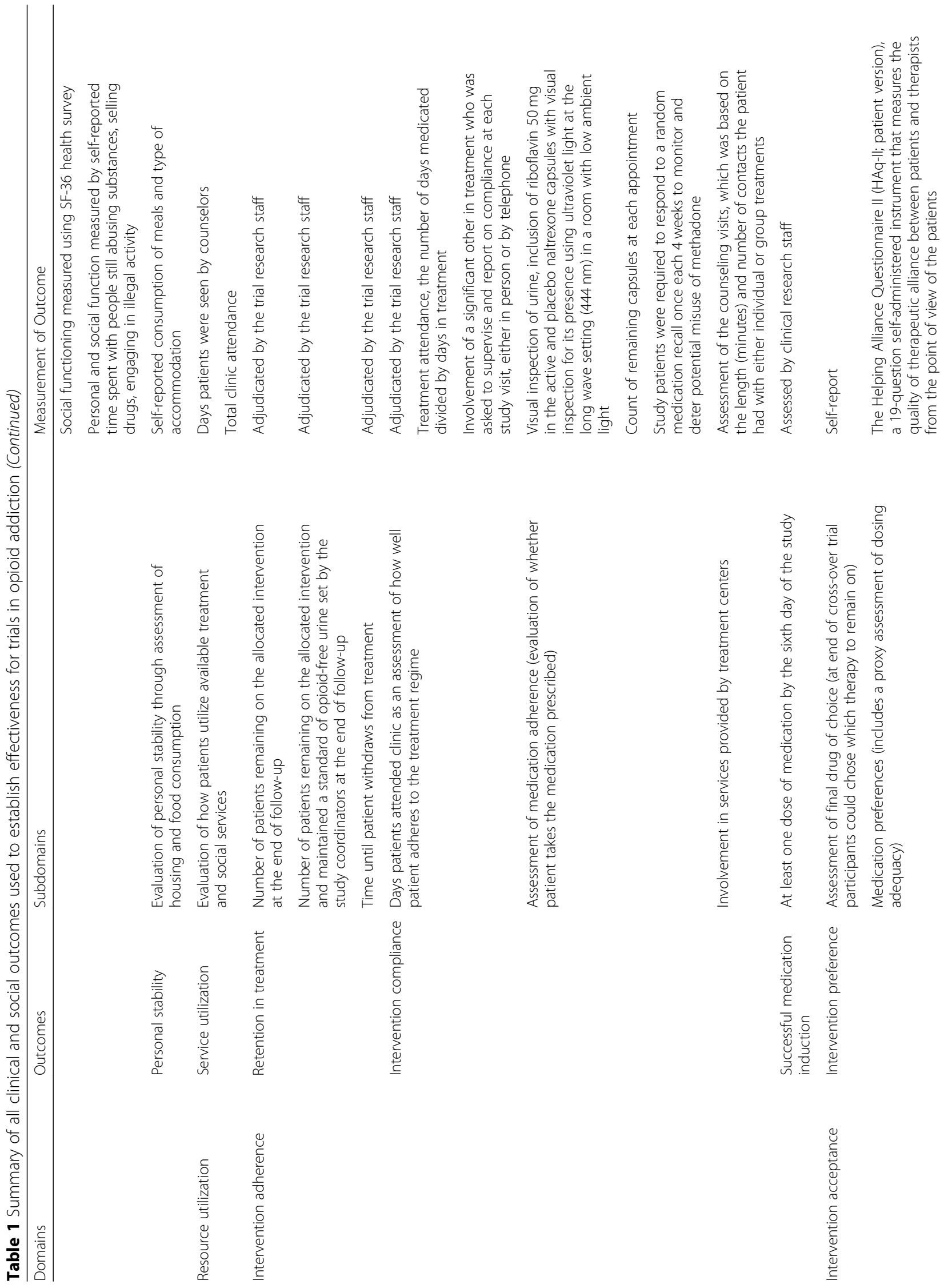




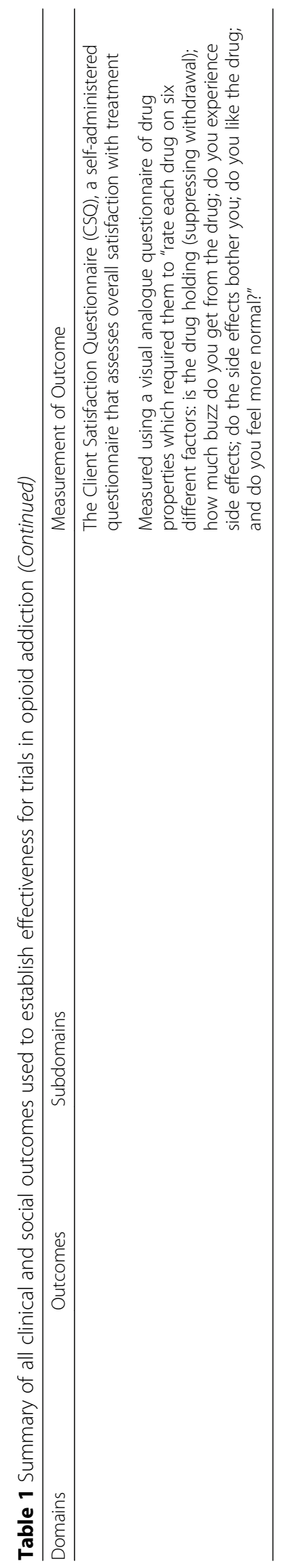


Table 2 Verbatim answers to qualitative interview to understand goals of therapy

\begin{tabular}{ll}
\hline Participant & Verbal answer \\
\hline 1 & Remain abstinent from drugs \\
3 & I don't want to use drugs \\
4 & Not use street drugs \\
5 & Get off opioids completely \\
6 & Maintain my job \\
7 & Just get my life back; I'm still an addict a \\
8 & nd I don't want that to sneak back on me \\
9 & To not be sick anymore \\
& Being completely off drugs. To never touch \\
drugs again
\end{tabular}

frequency of participants ranked goals of care was calculated and subsequently presented as a percentage.

Abstinence from opioid use was the most commonly selected outcome overall followed by stability of relationships, reduced money spent on drugs, reduced drug craving, employment, regaining physical health, pain control, coping, reduced depression, stable housing, improved sexual function, decreased risk of overdose, reduced injecting, and reduced anxiety overall across all participants' four outcome choices. The most commonly selected primary outcome for participants was abstinence from opioid use, with $47 \%$ (8) of participants selecting it as their first choice; $16.6 \%$ (3) chose money spent on drugs as their second most important outcome. Participant's outcomes are summarized in Table 3.

\section{Discussion}

Findings from this study outline the current outcomes employed in clinical trials for opioid addiction, and also provide a unique insight into the treatment goals patients consider important when receiving pharmacological therapies for OUD.

Results from the secondary review of outcome measures employed in OUD trials highlights a major lack of consensus in our evidence base when determining appropriate end-points for establishing treatment effectiveness for OSATs. A substantial number of outcomes as well as variations in the definitions and measurements of the same outcomes were reported across trials. Despite the overwhelming collection of outcomes employed by trialists, substance use-specifically opioid-and treatment retention remain the most consistently reported. Trialists seldom explored pharmacological effect on personal and social functioning outcomes such as criminal behavior, employment, relationships, and personal stability endpoints, including type of accommodation $(20 \%, n=12)$.

The most commonly employed outcomes used to establish effectiveness were in stark contrast to the goals for treatment patients described in the qualitative interviews performed for the second phase of our study. While the majority of patients agreed that abstinence and reduction in opioid use was a primary goal in their treatment, they also stressed goals for therapy comprising employment, improved relationship stability, reduction in the money spent on drugs, as well as improvements in physical and

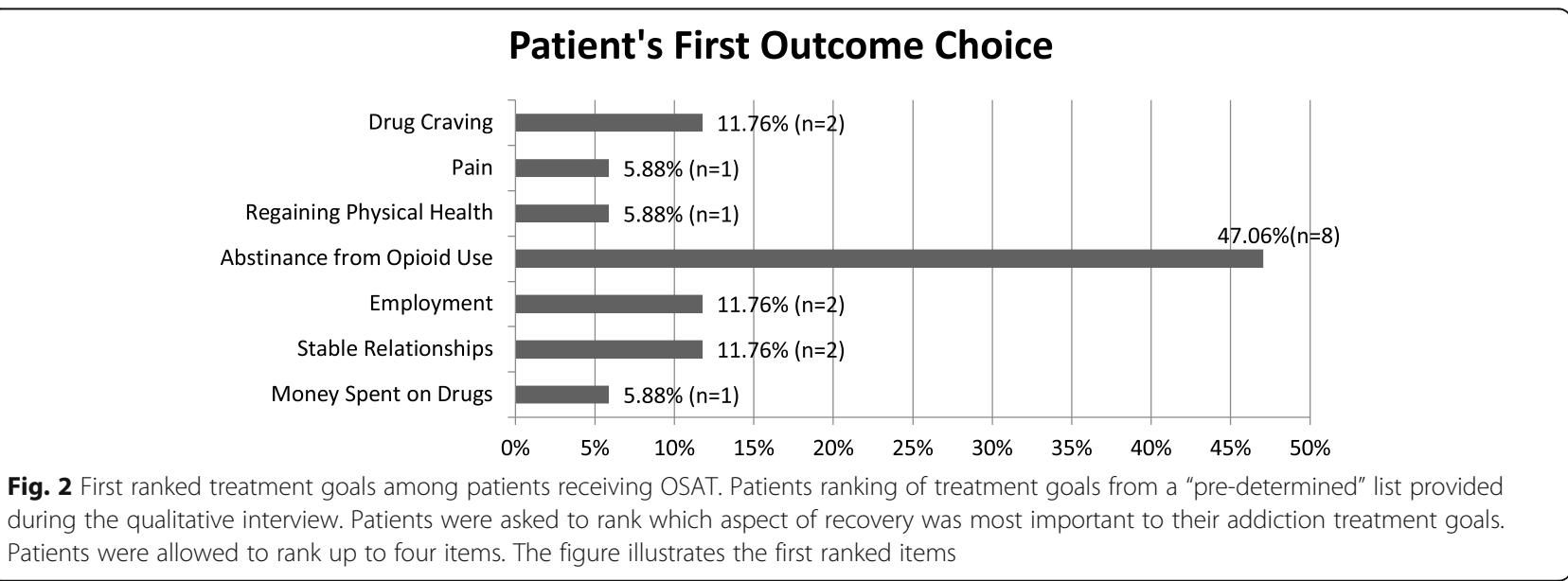


Table 3 Patients' responses to predetermined treatment goals

\begin{tabular}{|c|c|c|c|c|}
\hline Participant & Outcome 1 & Outcome 2 & Outcome 3 & Outcome 4 \\
\hline 1 & Money spent on drugs & Overdose & Injecting & NA \\
\hline 2 & Stable relationships & Coping & NA & NA \\
\hline 3 & Employment & Housing & Depression & \\
\hline 4 & Stable relationships & Money spent on drugs & Sexual function & Money spent on drugs \\
\hline 5 & Employment & Stable relationships & Housing & NA \\
\hline 6 & Abstinence from opioid use & Employment & NA & NA \\
\hline 7 & Regaining physical health & Abstinence from opioid use & NA & NA \\
\hline 8 & Abstinence from opioid us & Regaining physical health & Coping & NA \\
\hline 9 & Missing data & & & \\
\hline 10 & Abstinence from opioid use & Depression & Coping & NA \\
\hline 11 & Abstinence from opioid use & Drug craving & Money spent on drugs & Regaining physical health \\
\hline 12 & Pain & Employment & NA & NA \\
\hline 13 & Abstinence from opioid use & Money spent on drugs & Drug craving & Stable relationships \\
\hline 14 & Drug craving & Stable relationship & Money spent on drugs & NA \\
\hline 15 & Drug craving & Pain & & \\
\hline 16 & Abstinence from opioid use & Pain & Stable relationships & Drug craving \\
\hline 17 & Abstinence from opioid use & Money spent on drugs & Depression & Anxiety \\
\hline 18 & Abstinence from opioid use & Drug craving & Stable relationships & NA \\
\hline
\end{tabular}

NA not available

psychiatric symptoms such as pain, depression, and anxiety. Regrettably, these outcomes were rarely reported or of primary focus in the clinical trials. When assessing the comparative effectiveness of all interventions among patients receiving OSATs, retention in treatment was the most consistently measured and reported outcome across trials $(46 \%, n=28)$. In direct contrast to staying on treatment, our interviews with patients demonstrate an eagerness to complete therapy and get off the methadone treatment regime as a recurrent theme.

Outcome selection bears serious implications for the interpretation of the results as well as our ability to extrapolate such findings in a wider clinical context. These methodological shortcomings highlight the need for new assessment strategies for opioid addiction treatment options, where future efforts should consider targeting the objective assessment of treatment effectiveness employing long-term follow-up using administrative data-linkage for trial participants to evaluate hard long-term outcomes such as incidence of hepatitis, HIV, cardiovascular abnormalities, and mortality. Among the trials included in this review, three evaluated the impact of interventions on mortality [41, 60] or cardiac function [44].

Trials evaluating OSATs suffer from poor methodological quality [73]. A combination of small sample size, poor design, highly stringent eligibility criteria, effect estimates with tremendous imprecision, short-follow up time, missing data, and a major lack of consensus over patientimportant outcomes has led to an accumulation of a large yet very weak body of evidence. Whether it be illicit opioid use or risky behavior, the large number of definitions and measurements used to assess the same attribute suggest the need for more consensus in the field and understanding of what treatment outcomes are most important to addiction patients.

The evidence generated for this review was gathered from our previous work which aimed to determine the most effective pharmacotherapy for opioid use disorder [7]. An important finding from our original included the lack of standardization in outcome selection, in addition to the overall absence of discourse on patient-important outcomes in opioid use disorder. We felt strongly that this topic required a thorough discussion in a standalone paper and would be further complemented by the addition of qualitative interviews establishing patient values and preferences. We acknowledge the limitations posed by not updating our search strategy for the current study, particularly the lack of representation of studies conducted since the onset of the opioid crisis. However, our emphasis remains not establishing a superior therapy for addiction, which would require the most up-to-date assessment of all evidence, but rather to provide a summary of the outcome measures employed across clinical trials comprising the main body of evidence, which is largely captured in our current review, and likely would remain unchanged.

Efforts to map the health values and preferences of these 18 participants across all outcomes identified in the 
systematic review would have provided unique perspective to our current evaluation of the evidence. We hesitated to perform this analysis in light of the small sample size and absence of full representation of the outcome domains and subdomains identified from our review in the interview tool. Thus, any effort to draw conclusions regarding the representation of patient values in trial outcome selection could be explained by our lack of representation of the full list of trial outcomes in the interview tool.

Involvement of participants from our qualitative study phase in order to obtain a group consensus of the most valued goals of care would have been an instrumental addition to our evaluation of current OUD outcomes. Unfortunately, we did not hold ethics approval for that type of focus group work. It is clear a core outcomes set is needed in the field of OUD, which will require a larger, more representative study of all stakeholders. We maintain the key objectives of this work were to generate a discourse for patient-important outcomes in the OUD literature, and ultimately to provide the foundation for future researchers to explore this question in a larger representative sample.

\section{Conclusions}

In agreement with current guidelines, our study demonstrates there is limited consistency in the outcomes used to evaluate the effectiveness of OSATs [74, 75]. More concerning, our treatment recommendations and clinical decisions are being guided by a standard of effect considered useful to researchers yet in direct conflict with what patients deem important. This is a substantial limitation in the literature. Without the identification of a measurable treatment outcome that has an impact on and significance to patients, services, and the population as a whole, all the investment in trials will result in inadequate and inconsistent "efficacy" with limited, if any, external validity. We demonstrate here the need for an established set of OSAT outcomes guided by all stakeholders to inform clinicians of the true efficacy of these therapies and guide trialists to ensure our future understanding of these treatments accurately reflects the priorities of our patient population.

\section{Supplementary information}

Supplementary information accompanies this paper at https://doi.org/10 1186/s13063-019-3995-y.

Additional file 1. Interview tool: the interview tool used in qualitative interviews. Table S1. Summary of included trials: table summarizing important information from all trials included in this systematic review, including the journal, number of participants, and Cochrane risk of bias score.

\section{Abbreviations}

GENOA: Genetics of opioid addiction; HiREB: Hamilton Integrated Research Ethics Board; OSATs: Opioid substitution and antagonist therapies;
OUD: Opioid use disorder; PRISMA: Preferred reporting items for systematic reviews and meta-analyses; RCT: Randomized controlled trial

\section{Acknowledgements}

We would like to sincerely thank everyone who contributed to the completion of this project. We would like to specially thank Arnav Agarwal and Joshua Kong for their initial help with the screening and data abstraction of studies. This project would not have been possible without the great collaboration cemented between GENOA and the CATC.

\section{Authors' contributions}

$\mathrm{ZS}, \mathrm{BBD}$, and $\mathrm{LT}$ led the development of the project. BBD, NS, MB, LN, CP, $A W, J W, A B, N B M, A H, K C, B H, D R, P T, Z S$, and TB contributed to the development of the research protocol, which is published in the journal Systematic Reviews. BBD, NS, MB, LN, CP, AW, JW, AB, NBM, AH, KC, BH, DR, $\mathrm{PT}, \mathrm{ZS}$, and TB contributed to interpreting the data and writing the manuscript. ZS had full access to data from this investigation and she is accountable for the reliability of the data and the accuracy of all analyses performed. All authors read and approved the final manuscript.

\section{Funding}

This work was supported by a CIHR Drug Safety and Effectiveness Network (DSEN) grant (grant number 126639) and CIHR (sponsor award number

156306). The funders had no role in study design and conduct of the study; collection, management, analysis, and interpretation of the data; and preparation, review, or approval of the manuscript.

Availability of data and materials

The datasets used and/or analyzed during the current study are available from the corresponding author on reasonable request.

Ethics approval and consent to participate

Participants were recruited as part of the Genetics of Opioid Addiciton (GENOA) research collaborative between McMaster University and the Population Genomics Program. GENOA is a prospective cohort investigation approved by the Hamilton Integrated Research Ethics Board. Amendments to the GENOA study protocol to include new questions as part of our interview tool to allow for qualitative interviews exploring patient important outcomes was approved (HIREB \#0168).

\section{Consent for publication}

This study does not include any individual person's data requiring consent for publication.

\section{Competing interests}

The authors declare that they have no competing interests.

\section{Author details}

${ }^{1}$ McMaster University Internal Medicine Residency Program, Department of Medicine, Faculty of Health Sciences, McMaster University, Hamilton, Canada. ${ }^{2}$ Department of Health Research Methods, Evidence, and Impact, McMaster University, Hamilton, Canada. ${ }^{3}$ Department of Family Medicine Residency Program, Michael G. Degroote School of Medicine, McMaster University, Hamilton, Canada. ${ }^{4}$ Department of Psychiatry and Behavioural Neurosciences, McMaster University, 1280 Main Street West, Hamilton, Ontario L8S 4L8, Canada. ${ }^{5}$ Department of Medicine, Hamilton General Hospital, Hamilton, Canada. ${ }^{6}$ University of Toronto Faculty of Medicine, University of Toronto, Toronto, Canada. ${ }^{7}$ Faculty of Science, Department of Psychology, McGill University, Montreal, Canada. ${ }^{8}$ Center for Practice Changing Research, Ottawa Hospital Research Institute, Ottawa, Canada. ${ }^{9}$ Faculty of Medicine, University of Ottawa, Ottawa, ON, Canada. ${ }^{10}$ University of Ottawa Institute of Mental Health Research, Ottawa, ON, Canada. ${ }^{11}$ Clinical Epidemiology Program, Ottawa Hospital Research Institute, Ottawa, Ontario, Canada. ${ }^{12} \mathrm{~S}$ chool of Epidemiology and Public Health, University of Ottawa, Ottawa, Ontario, Canada. ${ }^{13}$ WHO Collaborating Centre for Knowledge Translation and Health Technology Assessment in Health Equity, Bruyère Research Institute, Ottawa, Canada. ${ }^{14}$ Centre for Evaluation of Medicine, Hamilton, Canada. ${ }^{15}$ System Linked Research Unit, Hamilton, Canada. ${ }^{16}$ Population Genomics Program, Chanchlani Research Center, McMaster University, Hamilton, Canada. 


\section{Received: 9 February 2019 Accepted: 13 December 2019}

\section{Published online: 06 January 2020}

\section{References}

1. Deshpande PR, Rajan S, Sudeepthi BL, Abdul Nazir CP. Patient-reported outcomes: a new era in clinical research. Perspect Clin Res. 2011:2:137-44.

2. Montori VM, Wang YG, Alonso-Coello P, Bhagra S. Systematic evaluation of the quality of randomized controlled trials in diabetes. Diabetes Care. 2006; 29:1833-8.

3. American Psychiatric Association. Diagnostic and statistical manual of mental disorders (5th ed.). 2013. https://doi.org/10.1176/appi.books. 9780890425596.

4. Mattick Richard P, Breen C, Kimber J, Davoli M. Methadone maintenance therapy versus no opioid replacement therapy for opioid dependence. Cochrane Database Syst Rev. 2009. https://doi.org/10.1002/14651858. CD002209.pub2

5. Hedrich D, et al. The effectiveness of opioid maintenance treatment in prison settings: a systematic review. Addiction. 2012;107:501-17.

6. Li Y, Kantelip J-P, Gerritsen-van Schieveen P, Davani S. Interindividual variability of methadone response. Mol Diagn Ther. 2008;12:109-24.

7. Dennis BB, Naji L, Bawor M, Bonner A, Varenbut M, Daiter J, Plater C, Pare G, Marsh DC, Worster A, Desai D. The effectiveness of opioid substitution treatments for patients with opioid dependence: a systematic review and multiple treatment comparison protocol. Syst Rev. 2014;3:105.

8. Shamseer $L$, et al. Preferred reporting items for systematic review and metaanalysis protocols (prisma-p) 2015: Elaboration and explanation. BMJ. 2015; 349:g7647.

9. McLellan AT, et al. The fifth edition of the Addiction Severity Index. J Subst Abus Treat. 1992;9:199-213.

10. Marsden J, Gossop G, Stewart D, Best D, Farrell M, Lehmann P, Edwards C, Strang J. The Maudsley Addiction Profile (MAP): A brief instrument for assessing treatment outcome. Addiction. 1998;93(12):1857-67.

11. Woo J, et al. "Dont judge a book by its cover": a qualitative study of methadone patients' experiences of stigma. Subst Abus Res Treat. 2017. https://doi.org/10.1177/1178221816685087.

12. von Elm E, et al. Strengthening the Reporting of Observational Studies in Epidemiology (STROBE) statement: guidelines for reporting observational studies. BMJ. 2007;335:806-8.

13. Ahmadi J, Ahmadi K. Controlled trial of maintenance treatment of intravenous buprenorphine dependence. Ir J Med Sci. 2003;172:171-3.

14. van den Brink W, et al. Medical prescription of heroin to treatment resistant heroin addicts: two randomised controlled trials. BMJ. 2003;327:310.

15. Ling W, Wesson DR, Charuvastra C, Klett CJ. A controlled trial comparing buprenorphine and methadone maintenance in opioid dependence. Arch Gen Psychiatry. 1996;53:401-7.

16. Schottenfeld RS, Chawarski MC, Mazlan M. Maintenance treatment with buprenorphine and naltrexone for heroin dependence in Malaysia: a randomised, double-blind, placebo-controlled trial. Lancet. 2008;371:2192-200.

17. Johnson RE, et al. A placebo controlled clinical trial of buprenorphine as a treatment for opioid dependence. Drug Alcohol Depend. 1995;40:17-25.

18. Ahmadi J, Moosavinasab M, Babaee M, Firoozabadi A, Mohagheghzadeh M, et al. F. H. Treatment of heroin dependence. Ger J Psychiatry. 2004;7:1-5.

19. Ahmadi J. A controlled trial of buprenorphine treatment for opium dependence: the first experience from Iran. Drug Alcohol Depend. 2002;66:111-4.

20. Yancovitz SR, et al. A randomized trial of an interim methadone maintenance clinic. Am J Public Heal. 1991;81:1185-91.

21. Sees KL, et al. Methadone maintenance vs 180-day psychosocially enriched detoxification for treatment of opioid dependence: a randomized controlled trial. JAMA. 2000;283:1303-10.

22. Strain EC, Stitzer ML, Liebson IA, Bigelow GE. Comparison of buprenorphine and methadone in the treatment of opioid dependence. Am J Psychiatry. 1994;151:1025-30

23. Soyka M, Zingg C, Koller G, Kuefner $H$. Retention rate and substance use in methadone and buprenorphine maintenance therapy and predictors of outcome: results from a randomized study. Int J Neuropsychopharmacol. 2008;11:641-53.

24. March JC, Oviedo-Joekes E, Perea-Milla E, Carrasco F. Controlled trial of prescribed heroin in the treatment of opioid addiction. J Subst Abus Treat. 2006:31:203-11.

25. Comer SD, et al. Injectable, sustained-release naltrexone for the treatment of opioid dependence: a randomized, placebo-controlled trial. Arch Gen Psychiatry. 2006;63:210-8
26. Eder $\mathrm{H}$, et al. Comparative study of the effectiveness of slow-release morphine and methadone for opioid maintenance therapy. Addiction (Abingdon, England). 2005;100:1101-9.

27. Eissenberg T, et al. Dose-related efficacy of levomethadyl acetate for treatment of opioid dependence. A randomized clinical trial. JAMA. 1997; 277:1945-51.

28. Jaffe $\mathrm{JH}$, et al. Methadyl acetate vs methadone. A double-blind study in heroin users. JAMA. 1972;222:437-42

29. Kamien JB, Branstetter SA, Amass L. Buprenorphine-naloxone versus methadone maintenance therapy: A randomised double-blind trial with opioid-dependent patients. Heroin Addict Relat Clin Probl. 2008;10:5-18.

30. King VL, et al. A 12-month controlled trial of methadone medical maintenance integrated into an adaptive treatment model. J Subst Abus Treat. 2006;31:385-93.

31. Oviedo-Joekes $E$, et al. Diacetylmorphine versus methadone for the treatment of opioid addiction. N Engl J Med. 2009;361:777-86.

32. Oviedo-Joekes E, March JC, Romero M, Perea-Milla E. The Andalusian trial on heroin-assisted treatment: a 2 year follow-up. Drug Alcohol Rev. 2010;29:75-80.

33. Potter JS, et al. Buprenorphine/naloxone and methadone maintenance treatment outcomes for opioid analgesic, heroin, and combined users: findings from starting treatment with agonist replacement therapies (STAR T). J Stud Alcohol Drugs. 2013;74:605-13.

34. Robertson JR, et al. Addressing the efficacy of dihydrocodeine versus methadone as an alternative maintenance treatment for opiate dependence: A randomized controlled trial. Addiction. 2006;101:1752-9.

35. Saxon AJ, et al. Buprenorphine/Naloxone and methadone effects on laboratory indices of liver health: a randomized trial. Drug Alcohol Depend. 2013;128:71-6.

36. Anglin MD, Conner BT, Annon J, Longshore D. Levo-alpha-acetylmethadol (LAAM) versus methadone maintenance: 1-year treatment retention, outcomes and status. Addiction. 2007;102:1432-42.

37. Schwartz RP, Kelly SM, O'Grady KE, Gandhi D, Jaffe JH. Interim methadone treatment compared to standard methadone treatment: 4-month findings. J Subst Abus Treat. 2011;41:21-9.

38. Schwartz RP, et al. A randomized controlled trial of interim methadone maintenance. Arch Gen Psychiatry. 2006;63:102-9.

39. Strain EC, Stitzer ML, Liebson IA, Bigelow GE. Buprenorphine versus methadone in the treatment of opioid dependence: self-reports, urinalysis, and addiction severity index. J Clin Psychopharmacol. 1996;16:58-67.

40. Wolstein J, et al. A randomized, open-label trial comparing methadone and Levo-Alpha-Acetylmethadol (LAAM) in maintenance treatment of opioid addiction. Pharmacopsychiatry. 2009:42:1-8.

41. Ling W, Charuvastra VC, Kaim SC, Klett CJ. Methadyl acetate and methadone as maintenance treatments for heroin addicts: a Veterans Administration cooperative study. Arch Gen Psychiatry. 1976;33:709-20.

42. White JM, et al. Relationship between LAAM-methadone preference and treatment outcomes. Drug Alcohol Depend. 2002;66:295-301.

43. Kosten TR, Schottenfeld R, Ziedonis D, Falcioni J. Buprenorphine versus methadone maintenance for opioid dependence. J Nerv Ment Dis. 1993; 181:358-64.

44. Wedam EF, Bigelow GE, Johnson RE, Nuzzo PA, Haigney MCP. QT-interval effects of methadone, levomethadyl, and buprenorphine in a randomized trial. Arch Intern Med. 2007;167:2469-75.

45. Woody GE, et al. Extended vs short-term buprenorphine-naloxone for treatment of opioid-addicted youth: a randomized trial. JAMA. 2008;300:2003-11.

46. Kakko J, Svanborg KD, Kreek MJ, Heilig M. 1-year retention and social function after buprenorphine-assisted relapse prevention treatment for heroin dependence in Sweden: a randomised, placebo-controlled trial. Lancet. 2003:361:662-8.

47. Fischer $\mathrm{G}$, et al. Buprenorphine versus methadone maintenance for the treatment of opioid dependence. Addiction (Abingdon, England). 1999;94: 1337-47.

48. Krook AL, et al. A placebo-controlled study of high dose buprenorphine in opiate dependents waiting for medication-assisted rehabilitation in Oslo, Norway. Addiction. 2002;97:533-42.

49. Ling $W$, et al. Buprenorphine maintenance treatment of opiate dependence: a multicenter, randomized clinical trial. Addiction. 1998;93:475-86.

50. Lintzeris $\mathrm{N}$, et al. Implementing buprenorphine treatment in community settings in Australia: experiences from the Buprenorphine Implementation Trial. Am J Addict. 2004;13(Suppl 1):S29-41. 
51. Mattick RP, et al. Buprenorphine versus methadone maintenance therapy: a randomized double-blind trial with 405 opioid-dependent patients. Addiction. 2003;98:441-52.

52. Neri S, et al. Randomized clinical trial to compare the effects of methadone and buprenorphine on the immune system in drug abusers. Psychopharmacology. 2005;179:700-4.

53. Shufman EN, et al. The efficacy of naltrexone in preventing reabuse of heroin after detoxification. Biol Psychiatry. 1994;35:935-45.

54. Pani PP, Maremmani I, Pirastu R, Tagliamonte A, Gessa GL. Buprenorphine: a controlled clinical trial in the treatment of opioid dependence. Drug Alcohol Depend. 2000;60:39-50.

55. Schottenfeld RS, Pakes JR, Kosten TR. Prognostic factors in Buprenorphineversus methadone-maintained patients. J Nerv Ment Dis. 1998;186:35-43.

56. Preston $\mathrm{KL}$, Umbricht A, Epstein $\mathrm{DH}$. Methadone dose increase and abstinence reinforcement for treatment of continued heroin use during methadone maintenance. Arch Gen Psychiatry. 2000;57:395-404.

57. Schottenfeld RS, et al. Methadone versus buprenorphine with contingency management or performance feedback for cocaine and opioid dependence. Am J Psychiatry. 2005;162:340-9.

58. Fudala PJ, et al. Office-based treatment of opiate addiction with a sublingual-tablet formulation of buprenorphine and naloxone. N Engl J Med. 2003;349:949-58.

59. Strain EC, Stitzer ML, Liebson IA, Bigelow GE. Dose-response effects of methadone in the treatment of opioid dependence. Ann Intern Med. 1993; 119:23-7.

60. Hartnoll RL, et al. Evaluation of heroin maintenance in controlled trial. Arch Gen Psychiatry. 1980;37:877-84.

61. Strang J, et al. Supervised injectable heroin or injectable methadone versus optimised oral methadone as treatment for chronic heroin addicts in England after persistent failure in orthodox treatment (RIOTT): a randomised trial. Lancet. 2010;375:1885-95.

62. Zaks A, Fink M, Freedman AM. Levomethadyl in maintenance treatment of opiate dependence. JAMA. 1972;220:811-3.

63. Strain EC, Bigelow GE, Liebson IA, Stitzer ML. Moderate- vs high-dose methadone in the treatment of opioid dependence: a randomized trial. JAMA. 1999;281:1000-5.

64. Petitjean $\mathrm{S}$, et al. Double-blind randomized trial of buprenorphine and methadone in opiate dependence. Drug Alcohol Depend. 2001;62:97-104.

65. Guo S. Efficacy of naltrexone hydrochloride for preventing relapse among opiate dependent patients after detoxification. Hong Kong J Psychiatry. 2001;11:2-8.

66. Krupitsky EM, et al. Naltrexone for heroin dependence treatment in St. Petersburg, Russia. J Subst Abus Treat. 2004;26:285-94.

67. San L, Pomarol G, Peri JM, Olle JM, Cami J. Follow-up after a six-month maintenance period on naltrexone versus placebo in heroin addicts. $\mathrm{Br} J$ Addict. 1991:86:983-90.

68. Krupitsky EM, et al. Naltrexone with or without fluoxetine for preventing relapse to heroin addiction in St. Petersburg, Russia. J Subst Abus Treat. 2006;31:319-28.

69. Giacomuzzi SM, Ertl M, Kemmler G, Riemer Y, Vigl A. Sublingual buprenorphine and methadone maintenance treatment: a three-year follow-up of quality of life assessment. Scientific World J. 2005;5:452-68.

70. Haasen $C$, et al. Heroin-assisted treatment for opioid dependence: randomised controlled trial. Br J Psychiatry. 2007;191:55-62.

71. Johnson RE, Jaffe JH, Fudala PJ. A controlled trial of buprenorphine treatment for opioid dependence. JAMA. 1992;267:2750-5.

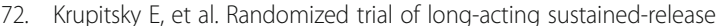
naltrexone implant vs oral naltrexone or placebo for preventing relapse to opioid dependence. Arch Gen Psychiatry. 2012;69:973-81.

73. Dennis BB, et al. Opioid substitution and antagonist therapy trials exclude the common addiction patient: a systematic review and analysis of eligibility criteria. Trials. 2015;16:1.

74. Bruneau J, et al. Management of opioid use disorders: a national clinical practice guideline. Can Med Assoc J. 2018;190:E247-57.

75. Methadone maintenance treatment program standards and clinical guidelines. The College of Physicians and Surgeons of Ontario; 2011.

\section{Publisher's Note}

Springer Nature remains neutral with regard to jurisdictional claims in published maps and institutional affiliations.

\section{Ready to submit your research? Choose BMC and benefit from:}

- fast, convenient online submission

- thorough peer review by experienced researchers in your field

- rapid publication on acceptance

- support for research data, including large and complex data types

- gold Open Access which fosters wider collaboration and increased citations

- maximum visibility for your research: over $100 \mathrm{M}$ website views per year

At $\mathrm{BMC}$, research is always in progress.

Learn more biomedcentral.com/submissions 